EUROPEAN LABORATORY FOR PARTICLE PHYSICS

CERN-PPE/96-104

$3^{\text {rd }}$ July 1996

\title{
Observation of charmless hadronic B decays
}

\author{
The ALEPH Collaboration
}

\begin{abstract}
Four candidates for charmless hadronic B decay are observed in a data sample of four million hadronic $\mathrm{Z}$ decays recorded by the ALEPH detector at LEP. The probability that these events come from background sources is estimated to be less than $10^{-6}$. The average branching ratio of weakly decaying $\mathrm{B}$ hadrons (a mixture of $\mathrm{B}_{\mathrm{d}}^{0}, \mathrm{~B}_{\mathrm{s}}^{0}$ and $\Lambda_{\mathrm{b}}$ weighted by their production cross sections and lifetimes, here denoted $B$ ) into two long-lived charged hadrons (pions, kaons or protons $)$ is measured to be $\mathcal{B} r\left(\mathrm{~B} \rightarrow \mathrm{h}^{+} \mathrm{h}^{-}\right)=\left(1.7_{-0.7}^{+1.0} \pm 0.2\right) \times 10^{-5}$. The relative branching fraction $\left(\mathcal{B} r\left(\mathrm{~B}_{\mathbf{d}}^{0} \rightarrow \pi^{+} \pi^{-}\right)+r_{\mathrm{s}} \mathcal{B} r\left(\mathrm{~B}_{\mathrm{s}}^{0} \rightarrow \pi^{+} \mathrm{K}^{-}\right)\right) /\left(\mathcal{B} r\left(\mathrm{~B}_{\mathbf{d}}^{0} \rightarrow \mathrm{h}^{+} \mathrm{h}^{-}\right)+r_{\mathrm{s}} \mathcal{B} r\left(\mathrm{~B}_{\mathrm{s}}^{0} \rightarrow \mathrm{h}^{+} \mathrm{h}^{-}\right)\right)$, where $r_{\mathrm{s}}$ is the ratio of $\mathrm{B}_{\mathrm{s}}^{0}$ to $\mathrm{B}_{\mathrm{d}}^{0}$ decays in the sample, is measured to be $1.0_{-0.3}^{+0.0}-_{-0.1}^{+0.0}$. In addition, branching ratio upper limits are obtained for a variety of exclusive charmless hadronic two-body decays of $B$ hadrons.
\end{abstract}

(To be submitted to Physics Letters B) 


\section{The ALEPH Collaboration}

D. Buskulic, I. De Bonis, D. Decamp, P. Ghez, C. Goy, J.-P. Lees, A. Lucotte, M.-N. Minard, J.-Y. Nief, P. Odier, B. Pietrzyk

Laboratoire de Physique des Particules (LAPP), IN ${ }^{2} P^{3}$-CNRS, 74019 Annecy-le-Vieux Cedex, France

M.P. Casado, M. Chmeissani, J.M. Crespo, M. Delfino, I. Efthymiopoulos ${ }^{1}$ E. Fernandez, M. Fernandez-Bosman, Ll. Garrido, ${ }^{15}$ A. Juste, M. Martinez, S. Orteu, C. Padilla, I.C. Park, A. Pascual, J.A. Perlas, I. Riu, F. Sanchez, F. Teubert Institut de Fisica d'Altes Energies, Universitat Autonoma de Barcelona, 08193 Bellaterra (Barcelona), Spain $^{7}$

A. Colaleo, D. Creanza, M. de Palma, G. Gelao, M. Girone, G. Taselli, G. Maggi, ${ }^{3}$ M. Maggi, N. Marinelli, S. Nuzzo, A. Ranieri, G. Raso, F. Ruggieri, G. Selvaggi, L. Silvestris, P. Tempesta, G. Zito

Dipartimento di Fisica, INFN Sezione di Bari, 70126 Bari, Italy

X. Huang, J. Lin, Q. Ouyang, T. Wang, Y. Xie, R. Xu, S. Xue, J. Zhang, L. Zhang, W. Zhao Institute of High-Energy Physics, Academia Sinica, Beijing, The People's Republic of China ${ }^{8}$

R. Alemany, A.O. Bazarko, G. Bonvicini ${ }^{23}$ P. Bright-Thomas, M. Cattaneo, P. Comas, P. Coyle, H. Drevermann, R.W. Forty, M. Frank, R. Hagelberg, J. Harvey, P. Janot, B. Jost, E. Kneringer, J. Knobloch, I. Lehraus, G. Lutters, E.B. Martin, P. Mato, A. Minten, R. Miquel, Ll.M. Mir, ${ }^{2}$ L. Moneta, T. Oest, ${ }^{20}$ A. Pacheco, J.-F. Pusztaszeri, F. Ranjard, P. Rensing, ${ }^{12}$ G. Rizzo, L. Rolandi, D. Schlatter, M. Schmelling, ${ }^{24}$ M. Schmitt, O. Schneider, W. Tejessy, I.R. Tomalin, A. Venturi, H. Wachsmuth, A. Wagner

European Laboratory for Particle Physics (CERN), 1211 Geneva 23, Switzerland

Z. Ajaltouni, A. Barrès, C. Boyer, A. Falvard, P. Gay, C . Guicheney, P. Henrard, J. Jousset, B. Michel, S. Monteil, J-C. Montret, D. Pallin, P. Perret, F. Podlyski, J. Proriol, P. Rosnet, J.-M. Rossignol

Laboratoire de Physique Corpusculaire, Université Blaise Pascal, $I N^{2} P^{3}-C N R S$, Clermont-Ferrand, 63177 Aubière, France

T. Fearnley, J.B. Hansen, J.D. Hansen, J.R. Hansen, P.H. Hansen, B.S. Nilsson, B. Rensch, A. Wäänänen Niels Bohr Institute, 2100 Copenhagen, Denmark ${ }^{9}$

A. Kyriakis, C. Markou, E. Simopoulou, I. Siotis, A. Vayaki, K. Zachariadou Nuclear Research Center Demokritos (NRCD), Athens, Greece

A. Blondel, G. Bonneaud, J.C. Brient, P. Bourdon, A. Rougé, M. Rumpf, A. Valassi ${ }^{6}{ }^{6}$ M. Verderi, H. Videau ${ }^{21}$ Laboratoire de Physique Nucléaire et des Hautes Energies, Ecole Polytechnique, $I N^{2} P^{3}$-CNRS, 91128 Palaiseau Cedex, France

D.J. Candlin, M.I. Parsons Department of Physics, University of Edinburgh, Edinburgh EH9 3JZ, United Kingdom ${ }^{10}$

E. Focardi, ${ }^{21}$ G. Parrini

Dipartimento di Fisica, Università di Firenze, INFN Sezione di Firenze, 50125 Firenze, Italy

M. Corden, C. Georgiopoulos, D.E. Jaffe Supercomputer Computations Research Institute, Florida State University, Tallahassee, FL 32306$4052, U_{S A}{ }^{13,14}$

A. Antonelli, G. Bencivenni, G. Bologna, ${ }^{4}$ F. Bossi, P. Campana, G. Capon, D. Casper, V. Chiarella, G. Felici,

P. Laurelli, G. Mannocchi, ${ }^{5}$ F. Murtas, G.P. Murtas, L. Passalacqua, M. Pepe-Altarelli Laboratori Nazionali dell'INFN (LNF-INFN), 00044 Frascati, Italy

L. Curtis, S.J. Dorris, A.W. Halley, I.G. Knowles, J.G. Lynch, V. O’Shea, C. Raine, P. Reeves, J.M. Scarr, K. Smith, P. Teixeira-Dias, A.S. Thompson, F. Thomson, S. Thorn, R.M. Turnbull Department of Physics and Astronomy, University of Glasgow, Glasgow G12 8QQ,United Kingdom ${ }^{10}$ 
U. Becker, C. Geweniger, G. Graefe, P. Hanke, G. Hansper, V. Hepp, E.E. Kluge, A. Putzer, M. Schmidt, J. Sommer,

H. Stenzel, K. Tittel, S. Werner, M. Wunsch

Institut für Hochenergiephysik, Universität Heidelberg, 69120 Heidelberg, Fed. Rep. of Germany ${ }^{16}$

D. Abbaneo, R. Beuselinck, D.M. Binnie, W. Cameron, P.J. Dornan, A. Moutoussi, J. Nash, J.K. Sedgbeer, A.M. Stacey, M.D. Williams

Department of Physics, Imperial College, London SW7 2BZ, United Kingdom ${ }^{10}$

G. Dissertori, P. Girtler, D. Kuhn, G. Rudolph

Institut für Experimentalphysik, Universität Innsbruck, 6020 Innsbruck, Austria ${ }^{18}$

A.P. Betteridge, C.K. Bowdery, P. Colrain, G. Crawford, A.J. Finch, F. Foster, G. Hughes, T. Sloan, M.I. Williams

Department of Physics, University of Lancaster, Lancaster LA1 4YB, United Kingdom ${ }^{10}$

A. Galla, I. Giehl, A.M. Greene, K. Jakobs, K. Kleinknecht, G. Quast, B. Renk, E. Rohne, H.-G. Sander, P. van Gemmeren C. Zeitnitz

Institut für Physik, Universität Mainz, 55099 Mainz, Fed. Rep. of Germany ${ }^{16}$

J.J. Aubert, ${ }^{21}$ A.M. Bencheikh, C. Benchouk, A. Bonissent, G. Bujosa, D. Calvet, J. Carr, C. Diaconu, F. Etienne, N. Konstantinidis, P. Payre, D. Rousseau, M. Talby, A. Sadouki, M. Thulasidas, K. Trabelsi

Centre de Physique des Particules, Faculté des Sciences de Luminy, IN ${ }^{2} P^{3}$-CNRS, 13288 Marseille, France

M. Aleppo, F. Ragusa ${ }^{21}$

Dipartimento di Fisica, Università di Milano e INFN Sezione di Milano, 20133 Milano, Italy

C. Bauer, R. Berlich, W. Blum, V. Büscher, H. Dietl, F. Dydak, ${ }^{21}$ G. Ganis, C. Gotzhein, H. Kroha, G. Lütjens,

G. Lutz, W. Männer, H.-G. Moser, R. Richter, A. Rosado-Schlosser, S. Schael, R. Settles, H. Seywerd, R. St. Denis,

H. Stenzel, W. Wiedenmann, G. Wolf

Max-Planck-Institut für Physik, Werner-Heisenberg-Institut, 80805 München, Fed. Rep. of Germany ${ }^{16}$

J. Boucrot, O. Callot, Y. Choi, ${ }^{26}$ A. Cordier, M. Davier, L. Duflot, J.-F. Grivaz, Ph. Heusse, A. Höcker, A. Jacholkowska, M. Jacquet, D.W. Kim ${ }^{19}$ F. Le Diberder, J. Lefrançois, A.-M. Lutz, I. Nikolic, H.J. Park, ${ }^{19}$

M.-H. Schune, S. Simion, J.-J. Veillet, I. Videau, D. Zerwas

Laboratoire de l'Accélérateur Linéaire, Université de Paris-Sud, $I N^{2} P^{3}$-CNRS, 91405 Orsay Cedex, France

P. Azzurri, G. Bagliesi, G. Batignani, S. Bettarini, C. Bozzi, G. Calderini, M. Carpinelli, M.A. Ciocci, V. Ciulli, R. Dell'Orso, R. Fantechi, I. Ferrante, L. Foà, ${ }^{1}$ F. Forti, A. Giassi, M.A. Giorgi, A. Gregorio, F. Ligabue, A. Lusiani, P.S. Marrocchesi, A. Messineo, F. Palla, G. Sanguinetti, A. Sciabà, P. Spagnolo, J. Steinberger, R. Tenchini, G. Tonelli, ${ }^{25}$ C. Vannini, P.G. Verdini, J. Walsh

Dipartimento di Fisica dell'Università, INFN Sezione di Pisa, e Scuola Normale Superiore, 56010 Pisa, Italy

G.A. Blair, L.M. Bryant, F. Cerutti, J.T. Chambers, Y. Gao, M.G. Green, T. Medcalf, P. Perrodo, J.A. Strong,

J.H. von Wimmersperg-Toeller

Department of Physics, Royal Holloway \& Bedford New College, University of London, Surrey TW20 OEX, United Kingdom ${ }^{10}$

D.R. Botterill, R.W. Clifft, T.R. Edgecock, S. Haywood, P. Maley, P.R. Norton, J.C. Thompson, A.E. Wright Particle Physics Dept., Rutherford Appleton Laboratory, Chilton, Didcot, Oxon OX11 OQX, United Kingdom $^{10}$

B. Bloch-Devaux, P. Colas, S. Emery, W. Kozanecki, E. Laņ̧on, M.C. Lemaire, E. Locci, B. Marx, P. Perez,

J. Rander, J.-F. Renardy, A. Roussarie, J.-P. Schuller, J. Schwindling, A. Trabelsi, B. Vallage CEA, DAPNIA/Service de Physique des Particules, CE-Saclay, 91191 Gif-sur-Yvette Cedex, France ${ }^{17}$

S.N. Black, J.H. Dann, R.P. Johnson, H.Y. Kim, A.M. Litke, M.A. McNeil, G. Taylor Institute for Particle Physics, University of California at Santa Cruz, Santa Cruz, CA 95064, USA ${ }^{22}$ 
C.N. Booth, R. Boswell, C.A.J. Brew, S. Cartwright, F. Combley, A. Koksal, M. Letho, W.M. Newton, J. Reeve, L.F. Thompson

Department of Physics, University of Sheffield, Sheffield S3 7RH, United Kingdom ${ }^{10}$

A. Böhrer, S. Brandt, G. Cowan, C. Grupen, J. Minguet-Rodriguez, F. Rivera, P. Saraiva, L. Smolik, F. Stephan, Fachbereich Physik, Universität Siegen, 57068 Siegen, Fed. Rep. of Germany ${ }^{16}$

M. Apollonio, L. Bosisio, R. Della Marina, G. Giannini, B. Gobbo, G. Musolino

Dipartimento di Fisica, Università di Trieste e INFN Sezione di Trieste, 34127 Trieste, Italy

J. Rothberg, S. Wasserbaech

Experimental Elementary Particle Physics, University of Washington, WA 98195 Seattle, U.S.A.

S.R. Armstrong, P. Elmer, Z. Feng, ${ }^{27}$ D.P.S. Ferguson, Y.S. Gao, ${ }^{28}$ S. González, J. Grahl, T.C. Greening, O.J. Hayes, H. Hu, P.A. McNamara III, J.M. Nachtman, W. Orejudos, Y.B. Pan, Y. Saadi, I.J. Scott, A.M. Walsh, ${ }^{29}$ Sau Lan Wu, X. Wu, J.M. Yamartino, M. Zheng, G. Zobernig

Department of Physics, University of Wisconsin, Madison, WI 53706, USA ${ }^{11}$

${ }^{1}$ Now at CERN, 1211 Geneva 23, Switzerland.

${ }^{2}$ Supported by Dirección General de Investigación Científica y Técnica, Spain.

${ }^{3}$ Now at Dipartimento di Fisica, Università di Lecce, 73100 Lecce, Italy.

${ }^{4}$ Also Istituto di Fisica Generale, Università di Torino, Torino, Italy.

${ }^{5}$ Also Istituto di Cosmo-Geofisica del C.N.R., Torino, Italy.

${ }^{6}$ Supported by the Commission of the European Communities, contract ERBCHBICT941234.

${ }^{7}$ Supported by CICYT, Spain.

${ }^{8}$ Supported by the National Science Foundation of China.

${ }^{9}$ Supported by the Danish Natural Science Research Council.

${ }^{10}$ Supported by the UK Particle Physics and Astronomy Research Council.

${ }^{11}$ Supported by the US Department of Energy, grant DE-FG0295-ER40896.

${ }^{12}$ Now at Dragon Systems, Newton, MA 02160, U.S.A.

${ }^{13}$ Supported by the US Department of Energy, contract DE-FG05-92ER40742.

${ }^{14}$ Supported by the US Department of Energy, contract DE-FC05-85ER250000.

${ }^{15}$ Permanent address: Universitat de Barcelona, 08208 Barcelona, Spain.

${ }^{16}$ Supported by the Bundesministerium für Forschung und Technologie, Fed. Rep. of Germany.

${ }^{17}$ Supported by the Direction des Sciences de la Matière, C.E.A.

${ }^{18}$ Supported by Fonds zur Förderung der wissenschaftlichen Forschung, Austria.

${ }^{19}$ Permanent address: Kangnung National University, Kangnung, Korea.

${ }^{20}$ Now at DESY, Hamburg, Germany.

${ }^{21}$ Also at CERN, 1211 Geneva 23, Switzerland.

${ }^{22}$ Supported by the US Department of Energy, grant DE-FG03-92ER40689.

${ }^{23}$ Now at Wayne State University, Detroit, MI 48202, USA.

${ }^{24}$ Now at Max-Plank-Institüt für Kernphysik, Heidelberg, Germany.

${ }^{25}$ Also at Istituto di Matematica e Fisica, Università di Sassari, Sassari, Italy.

${ }^{26}$ Permanent address: Sung Kyun Kwon University, Suwon, Korea.

${ }^{27}$ Now at The Johns Hopkins University, Baltimore, MD 21218, U.S.A.

${ }^{28}$ Now at Harvard University, Cambridge, MA 02138, U.S.A.

${ }^{29}$ Now at Rutgers University, Piscataway, NJ 08855-0849, U.S.A. 


\section{Introduction}

Decays of B hadrons into final states which do not contain a charm quark can proceed either via a tree-level $b \rightarrow \mathrm{u}$ transition or through one-loop penguin diagrams. For the decay ${ }^{1} \mathrm{~B}_{\mathrm{d}}^{0} \rightarrow \pi^{+} \pi^{-}$, the contribution coming from $\mathrm{b} \rightarrow \mathrm{u}$ transitions is expected to dominate [1]. The contribution originating from penguin diagrams is believed to be more important for the decay $\mathrm{B}_{\mathrm{d}}^{0} \rightarrow \mathrm{K}^{+} \pi^{-}[2]$. The decay $\mathrm{B}_{\mathrm{d}}^{0} \rightarrow \pi^{+} \pi^{-}$is of particular interest since it is expected to play an important role in future studies of $\mathrm{CP}$ violation in the $\mathrm{b}$ system [3]. The interpretation of such studies depends on the branching ratios for the channels $\mathrm{B}_{\mathbf{d}}^{0} \rightarrow \pi^{+} \pi^{-}$and $\mathrm{B}_{\mathbf{d}}^{0} \rightarrow \mathrm{K}^{+} \pi^{-}$as well as on the relative contributions of the $\mathrm{b} \rightarrow \mathrm{u}$ and penguin amplitudes.

The CLEO collaboration has found the first evidence for charmless hadronic B decays [4]. They measure a combined branching ratio $\mathcal{B} r\left(\mathrm{~B}_{\mathrm{d}}^{0} \rightarrow \pi^{+} \pi^{-}+\mathrm{K}^{+} \pi^{-}\right)=\left(1.8_{-0.5}^{+0.6}+0.2 \pm 0.2\right) \times 10^{-5}$ but are unable to obtain statistically significant signals in the two individual channels. Searches for such processes have recently been undertaken at LEP, where the B hadrons are heavily boosted and their decay products originate from distinct secondary vertices. This feature, when exploited by the use of high precision vertex detectors, allows an effective tagging of the decay products of the B hadrons. The OPAL collaboration has set branching ratio upper limits on a variety of two-body final states [5]. The DELPHI collaboration has observed five candidate events of the type $\mathrm{B} \rightarrow \mathrm{h}^{+} \mathrm{h}^{-}[6]$. In the ALEPH data sample of four million hadronic $\mathrm{Z}$ decays, collected between 1991 and 1995, the CLEO measurements would predict the production of approximately 13 decays of the type $\mathrm{B}_{\mathrm{d}}^{0} \rightarrow \pi^{+} \pi^{-}, \mathrm{K}^{+} \pi^{-}$. At LEP it is also possible to look for the corresponding charmless decays of both $B_{\mathrm{s}}^{0}$ and $\Lambda_{\mathrm{b}}$.

\section{The ALEPH detector}

The ALEPH detector and its performance are described in detail elsewhere $[7,8]$ and only a brief description is given here.

A high resolution vertex detector (VDET) consisting of two layers of double-sided silicon microstrip detectors surrounds the beam pipe. The inner layer is at an average radius of $6.5 \mathrm{~cm}$ from the beam axis and covers $85 \%$ of the solid angle, while the outer layer is at an average radius of $11.3 \mathrm{~cm}$ and covers $69 \%$. The spatial resolution for the $r \phi$ and $z$ projections (transverse to and along the beam direction, respectively) is $12 \mu \mathrm{m}$ at normal incidence. The vertex detector is surrounded by a drift chamber with eight axial wire layers and a time projection chamber (TPC) that measures up to 21 three-dimensional points per track. These detectors are immersed in an axial magnetic field of $1.5 \mathrm{~T}$ and together provide a momentum resolution of $\sigma_{p} / p=0.0006 p \oplus 0.005$ ( $p$ in $\mathrm{GeV} / c$ ). For tracks with hits in both layers of the VDET, the impact parameter resolution may be parameterized as $\sigma_{\delta}=25 \mu \mathrm{m}+95 \mu \mathrm{m} / p(p$ in $\mathrm{GeV} / c)$ in both the $r \phi$ and $r z$ views. The TPC also provides up to 338 measurements of the specific ionization of a charged particle $(\mathrm{dE} / \mathrm{dx})$. An average $\mathrm{K} / \pi$ separation of approximately two standard deviations is achieved for charged tracks with at least $50 \mathrm{dE} / \mathrm{dx}$ measurements and momenta greater than $3 \mathrm{GeV} / c$. The $\mathrm{dE} / \mathrm{dx}$ measurements are expressed as the difference between the measured and expected specific energy loss in units of standard deviations for a given particle mass hypothesis.

This tracking system is surrounded by a lead/wire-plane sampling calorimeter which is used to measure electromagnetic energy and to identify electrons and photons. The iron return yoke of

\footnotetext{
${ }^{1}$ Throughout this paper charge conjugate modes are always implied.
} 
the magnet is instrumented with streamer tubes to form a hadron calorimeter. It is surrounded by two additional double layers of streamer tubes which are used to provide muon identification.

\section{Event selection}

The selection of hadronic events is based on charged tracks and is described elsewhere [9]. For these events an interaction point is reconstructed on an event by event basis using the constraint of the beam axis position and the size of the luminous region [10]. This interaction point is determined with an average resolution of $85 \mu \mathrm{m}$ for $\mathrm{b} \bar{b}$ events, projected along the sphericity axis of the event. Charmless hadronic B decays are identified by looking for two oppositely charged particles, originating from a displaced vertex, with a mass above the kinematic limit for particles coming from B decays involving charm. Such decays are selected using a combination of cuts based on kinematics and vertex topology.

\section{Kinematics:}

- Each track is required to have a momentum above $3 \mathrm{GeV} / \mathrm{c}$.

- The momentum of the $\mathrm{B}$ hadron candidate has to be at least $30 \mathrm{GeV} / c$.

\section{Vertex topology:}

- Each track is required to have at least one $r \phi$ coordinate and at least one $z$ coordinate in the VDET.

- The three-dimensional impact parameter of each track has to be inconsistent with the interaction point by more than three standard deviations.

- Each track has to cross the flight path of the B hadron candidate downstream of the interaction point.

- The two tracks are required to form a vertex with a probability of at least $1 \%$.

- This vertex has to be at least six standard deviations and at most $2 \mathrm{~cm}$ downstream of the interaction point.

- The B hadron candidate is required to point back from its vertex to the interaction point with a probability of at least $1 \%$.

Additionally a track pair is rejected if either track is an identified lepton candidate [11].

Monte Carlo studies show that the maximum mass of two tracks coming from a B meson decay involving charm and passing the above kinematic cuts, with each track assigned the pion mass, is approximately $4.8 \mathrm{GeV} / c^{2}$. The corresponding limit from $\mathrm{B}$ baryon decays involving charm is $5.0 \mathrm{GeV} / c^{2}$. A signal region is defined by requiring that: (i) the mass of the two tracks, under the pion hypothesis, be at least three standard deviations above $5 \mathrm{GeV} / c^{2}$ and (ii) the mass of the two tracks be less than three standard deviations above the $\Lambda_{\mathrm{b}}$ mass [12] when one track is given the proton hypothesis. The average resolution on the measured mass obtained from a sample of $\mathrm{B}_{\mathrm{d}}^{0} \rightarrow \pi^{+} \pi^{-}$Monte Carlo events is $44 \mathrm{MeV} / c^{2}$. The resolution on the measured $\mathrm{B}$ hadron mass is studied in the data using a sample of cleanly reconstructed $\mathrm{J} / \psi \rightarrow \mu^{+} \mu^{-}$decays. A scale factor on the uncertainty on the mass of $1.20 \pm 0.05$ is estimated. This factor, which is applied to the mass 
uncertainty estimated by the reconstruction program, is taken into account in the above selection and is included in any quoted mass uncertainties.

The efficiency of the above selection criteria was determined using Monte Carlo simulation to be $(21.0 \pm 0.6) \%$ for the decay $\mathrm{B}_{\mathrm{d}}^{0} \rightarrow \pi^{+} \pi^{-}$, and $(20.4 \pm 0.6) \%$ for the decay $\Lambda_{\mathrm{b}} \rightarrow \mathrm{p} \pi^{-}$. It is also possible for charmless hadronic $B$ decays involving additional neutral particles to pass the above selection. The efficiency for one such decay, $\mathrm{B}_{\mathbf{d}}^{0} \rightarrow \pi^{ \pm} \rho^{\mp}$, was determined to be $(0.5 \pm 0.2) \%$.

\section{Results}

The above selection criteria were applied to a sample of four million hadronic $\mathrm{Z}$ decays. The mass distribution, under the pion hypothesis, is shown for both unlike-sign and like-sign pairs in Figure 1. In the unlike-sign pair sample there are four candidate events satisfying the selection criteria outlined above. Some relevant properties of these candidate events are given in Table 1. The four selected decays are all compatible with at least one two-body decay hypothesis $B \rightarrow h^{+} h^{-}$. There is one additional event above $5 \mathrm{GeV} / c^{2}$, with a measured mass of $5.08 \pm 0.06 \mathrm{GeV} / c^{2}$ and therefore less than three standard deviations above $5 \mathrm{GeV} / c^{2}$. In this event there is $1.2 \mathrm{GeV}$ of neutral electromagnetic energy near the $\mathrm{B}$ hadron candidate. If this energy deposition is interpreted as originating from a $\pi^{0}$ decay this event is consistent with a charmless hadronic $\mathrm{B}$ decay of the type $B_{d}^{0} \rightarrow \pi^{ \pm} \rho^{\mp}$. In the like-sign pair control sample there are no candidates with a mass, under the pion hypothesis, above $5 \mathrm{GeV} / c^{2}$. The four candidate events were investigated further to try and identify possible effects, such as pattern recognition mistakes, which could cause the vertex properties of the events to be mismeasured. No such problems were found. An event display of one of the four candidates is shown in Figure 2.

A background estimate for the above selection was obtained using a Monte Carlo sample of hadronic Z decays simulated without charmless B decays. Events were selected with slightly relaxed kinematic cuts at the generator level and were then passed through the full detector simulation and reconstruction. The ability of the Monte Carlo to reproduce accurately the level of background in the data was tested by removing some of the selection criteria. In particular, this was studied using like-sign track pairs which passed the kinematic cuts (with a relaxed momentum cut $\left.P_{B}>20 \mathrm{GeV} / c\right)$, had at least one $r \phi$ coordinate and at least one $z$ coordinate in the VDET and had a significant impact parameter. There should be no contribution from $\mathrm{B}$ hadron decays to these like-sign track pairs. In the mass region at least three standard deviations above 5.0 and below $6.5 \mathrm{GeV} / c^{2}$ there are 156 such track pairs observed in the data compared with a Monte Carlo prediction of $52 \pm 6$. This underestimate of the background in the Monte Carlo, by a factor of three, remains when both tracks are further required to cross the flight path of the $B$ hadron candidate downstream of the interaction point. The origin of this discrepancy is not fully understood, but a large fraction of it can be attributed to a previously observed excess of tracks with high transverse momentum relative to their jet axes observed in the data with respect to Monte Carlo predictions [13]. In the present analysis when only the kinematic cuts are applied the Monte Carlo underestimates the data by a factor of 1.6, which explains only a part of the effect. When normalised to the number of observed events, the Monte Carlo provides an adequate description of a variety of relevant variables for track pairs passing the relaxed selection (shown in Figure 3). Figure 3(a-c) indicates that the simulation provides an adequate description of the shape of both kinematic variables used in the selection and also of the tails of the impact parameter distribution. To study the flavour composition of the background the probability $\mathcal{P}_{\text {uds }}$ that all tracks in the hemisphere opposite to the $\mathrm{B}$ hadron candidate originate from the interaction point 


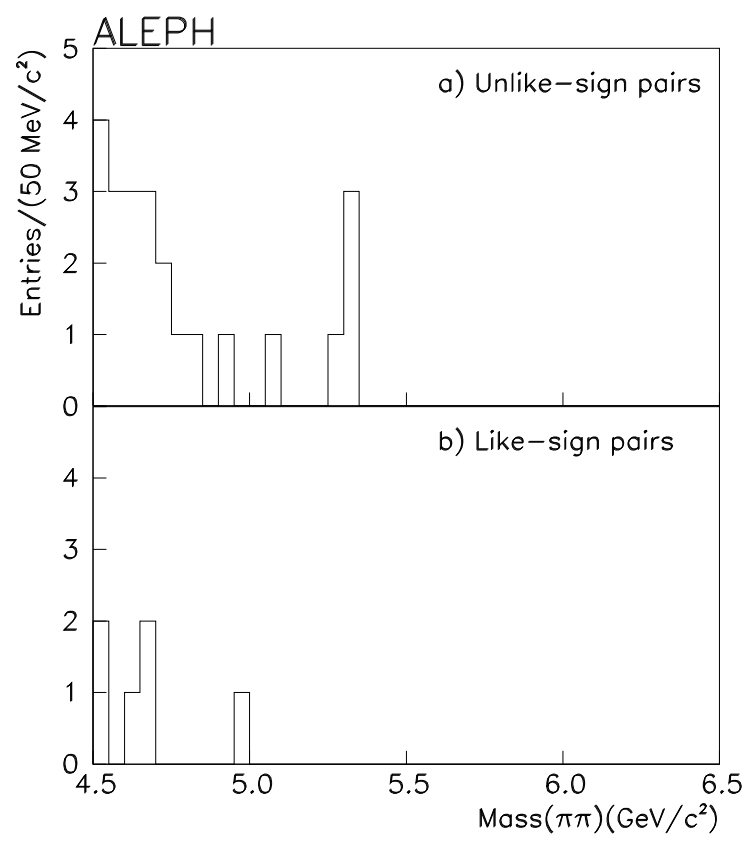

Figure 1: Mass distributions, under the pion hypothesis, for both (a) unlike-sign and (b) like-sign candidates.

was calculated [14]. From the distribution of $\mathcal{P}_{\text {uds }}$, shown in Figure 3(d), the relative proportion of background coming from $\mathrm{B}$ decays (which would preferentially have a low value of $\mathcal{P}_{\text {uds }}$ ) and from light quark decays is seen to be well reproduced by the Monte Carlo. (The values of $\mathcal{P}_{\text {uds }}$ obtained for the four candidate events are given in Table 1 . One event has a probability of $54 \%$. The probability of at least one out of four simulated signal events having a probability of this value or larger is $12 \%$.) To account for the above factor-of-three discrepancy, it is assumed that the probability to observe a background event in the data, after all selection criteria are applied, is three times higher than predicted by the Monte Carlo. It is further assumed that after the final cuts the background is flat in the above mass region, and that like-sign and unlike-sign background events are produced at equal rate. These final two assumptions are valid in data and Monte Carlo for the relaxed selection described above. A background estimate of $0.05 \pm 0.02$ was obtained from Monte Carlo simulation. To obtain sufficient statistics a preselection was applied at the generator level. This estimate takes into account the observed factor-of-three discrepancy in the background normalisation. The probability for observing four or more background events in the data in the signal region is less than $10^{-6}$.

\section{Branching ratio measurements}

From the number of selected events, the average branching ratio of weakly decaying $B$ hadrons into two long-lived charged hadrons (pions, kaons or protons) with no accompanying neutral particles is measured to be $\mathcal{B r}\left(\mathrm{B} \rightarrow \mathrm{h}^{+} \mathrm{h}^{-}\right)=\left(1.7_{-0.7}^{+1.0} \pm 0.2\right) \times 10^{-5}$. This measurement assumes that corresponding decays of $\mathrm{B}_{\mathrm{d}}^{0}, \mathrm{~B}_{\mathrm{s}}^{0}$ and $\Lambda_{\mathrm{b}}$ have equal partial widths $\left(\right.$ e.g., $\Gamma\left(\mathrm{B}_{\mathrm{d}}^{0} \rightarrow \pi^{+} \pi^{-}\right)=$ 
Table 1: Some properties of the four candidate events. For reference the masses of the $B_{d}^{0}$ and $B_{s}^{0}$ mesons were taken as $5.279 \pm 0.002$ and $5.375 \pm 0.006 \mathrm{GeV} / c^{2}$, respectively (PDG94).

\begin{tabular}{|l|c|c|c|c|}
\hline & Event a & Event b & Event c & Event $\mathrm{d}$ \\
\hline B candidate momentum $P_{B}(\mathrm{GeV} / c)$ & 38 & 40 & 35 & 39 \\
\hline Track momentum $P^{+}(\mathrm{GeV} / c)$ & 9 & 30 & 9 & 33 \\
\hline Track momentum $P^{-}(\mathrm{GeV} / c)$ & 30 & 10 & 26 & 7 \\
\hline Impact parameter significance $S^{+}$ & 38 & 27 & 44 & 5 \\
\hline Impact parameter significance $S^{-}$ & 15 & 68 & 25 & 22 \\
\hline Mass $M_{\pi^{+} \pi^{-}}\left(\mathrm{GeV} / c^{2}\right)$ & 5.337 & 5.338 & 5.285 & 5.349 \\
\hline Mass $M_{\pi^{+} K^{-}}\left(\mathrm{GeV} / c^{2}\right)$ & 5.364 & 5.419 & 5.313 & 5.469 \\
\hline Mass $M_{K^{+} \pi^{-}}\left(\mathrm{GeV} / c^{2}\right)$ & 5.427 & 5.366 & 5.367 & 5.375 \\
\hline Mass $M_{K^{+} K^{-}}\left(\mathrm{GeV} / c^{2}\right)$ & 5.454 & 5.447 & 5.395 & 5.494 \\
\hline Mass $M_{\mathrm{p} \pi^{-}}\left(\mathrm{GeV} / c^{2}\right)$ & 5.675 & 5.445 & 5.592 & 5.446 \\
\hline Mass $M_{\mathrm{p} K^{-}}\left(\mathrm{GeV} / c^{2}\right)$ & 5.700 & 5.525 & 5.619 & 5.564 \\
\hline Mass $M_{\pi^{+}}\left(\mathrm{GeV} / c^{2}\right)$ & 5.441 & 5.643 & 5.394 & 5.795 \\
\hline Mass $M_{K^{+}}\left(\mathrm{GeV} / c^{2}\right)$ & 5.529 & 5.670 & 5.474 & 5.819 \\
\hline Uncertainty on the mass $\left(\mathrm{GeV} / c^{2}\right)$ & 0.054 & 0.055 & 0.036 & 0.058 \\
\hline $\mathrm{dE} / \mathrm{dx} \sigma$ from $\pi^{+}$hypothesis & 0.1 & -2.3 & -0.6 & -1.3 \\
\hline $\mathrm{dE} / \mathrm{dx} \sigma$ from $K^{+}$hypothesis & 2.0 & -0.5 & 1.9 & 0.2 \\
\hline $\mathrm{dE} / \mathrm{dx} \sigma$ from $p$ hypothesis & 3.2 & 0.7 & 3.3 & 1.3 \\
\hline $\mathrm{dE} / \mathrm{dx} \sigma$ from $\pi^{-}$hypothesis & $\mathbf{0 . 5}$ & 0.7 & 0.6 & 2.0 \\
\hline $\mathrm{dE} / \mathrm{dx} \sigma$ from $K^{-}$hypothesis & 2.2 & 3.3 & 2.7 & 5.1 \\
\hline $\mathrm{dE} / \mathrm{dx} \sigma$ from $\bar{p}$ hypothesis & 3.4 & 5.0 & 4.3 & 6.9 \\
\hline Decay length $(\mathrm{cm})$ & $\mathbf{0 . 6 0}$ & $\mathbf{0 . 9 5}$ & $\mathbf{0 . 6 3}$ & $\mathbf{0 . 2 3}$ \\
\hline Decay length significance & 41 & 73 & 55 & 23 \\
\hline Proper decay time $(\mathrm{ps})$ & 4.8 & 3.2 & 1.1 \\
\hline $\mathcal{P}$ uds from opposite hemisphere & $\mathbf{0 . 5 4}$ & $10^{-3}$ & $10^{-3}$ \\
\hline
\end{tabular}

$\left.\Gamma\left(\mathrm{B}_{\mathrm{s}}^{0} \rightarrow \pi^{+} \mathrm{K}^{-}\right)=\Gamma\left(\Lambda_{\mathrm{b}} \rightarrow \mathrm{p} \pi^{-}\right)\right)$. The relevant ratios of branching ratios are obtained from this assumption on the partial widths by weighting with the measured lifetimes of the different $B$ hadrons [15] and the fractions of $\mathrm{B}_{\mathrm{d}}^{0}, \mathrm{~B}_{\mathrm{s}}^{0}$ or $\Lambda_{\mathrm{b}}$ resulting from the hadronization of a $\mathrm{b}$ quark taken to be $(37.8 \pm 2.2) \%,(11.2 \pm 1.9) \%$ and $(13.2 \pm 4.1) \%$, respectively [16]. They are in agreement with theoretical expectations $[2,17]$.

The systematic uncertainty quoted on the measured branching fraction contains contributions from the various sources listed in Table 2. The uncertainties in the hadronization of $b$ quarks into the different $\mathrm{B}$ hadrons and their measured lifetimes give a contribution of $2 \%$. The combinatorial background contribution is estimated to be $1 \%$ based on the above Monte Carlo prediction of 0.05 events expected. The contribution of charmless hadronic decays involving additional neutral particles is estimated using the ratio of the efficiency estimates obtained for the channels $\mathrm{B}_{\mathbf{d}}^{0} \rightarrow \pi^{+} \pi^{-}$and $\mathrm{B}_{\mathbf{d}}^{0} \rightarrow \pi^{ \pm} \rho^{\mp}$. The branching ratio $\mathcal{B} r\left(\mathrm{~B}_{\mathbf{d}}^{0} \rightarrow \pi^{ \pm} \rho^{\mp}\right)$ is taken to be three times greater than the branching ratio $\mathcal{B} r\left(\mathrm{~B}_{\mathbf{d}}^{0} \rightarrow \pi^{+} \pi^{-}\right)$, as expected from theoretical predictions [17]. The four selected decays have a mass inconsistent by more than three standard deviations with the $\mathrm{B}_{\mathbf{d}}^{0} \rightarrow \pi^{ \pm} \rho^{\mp}$ hypothesis. The $\mathrm{b}$ quark fragmentation function was varied within its experimental uncertainty [18] leading to a $3 \%$ change in efficiency. The ability of the Monte Carlo simulation 


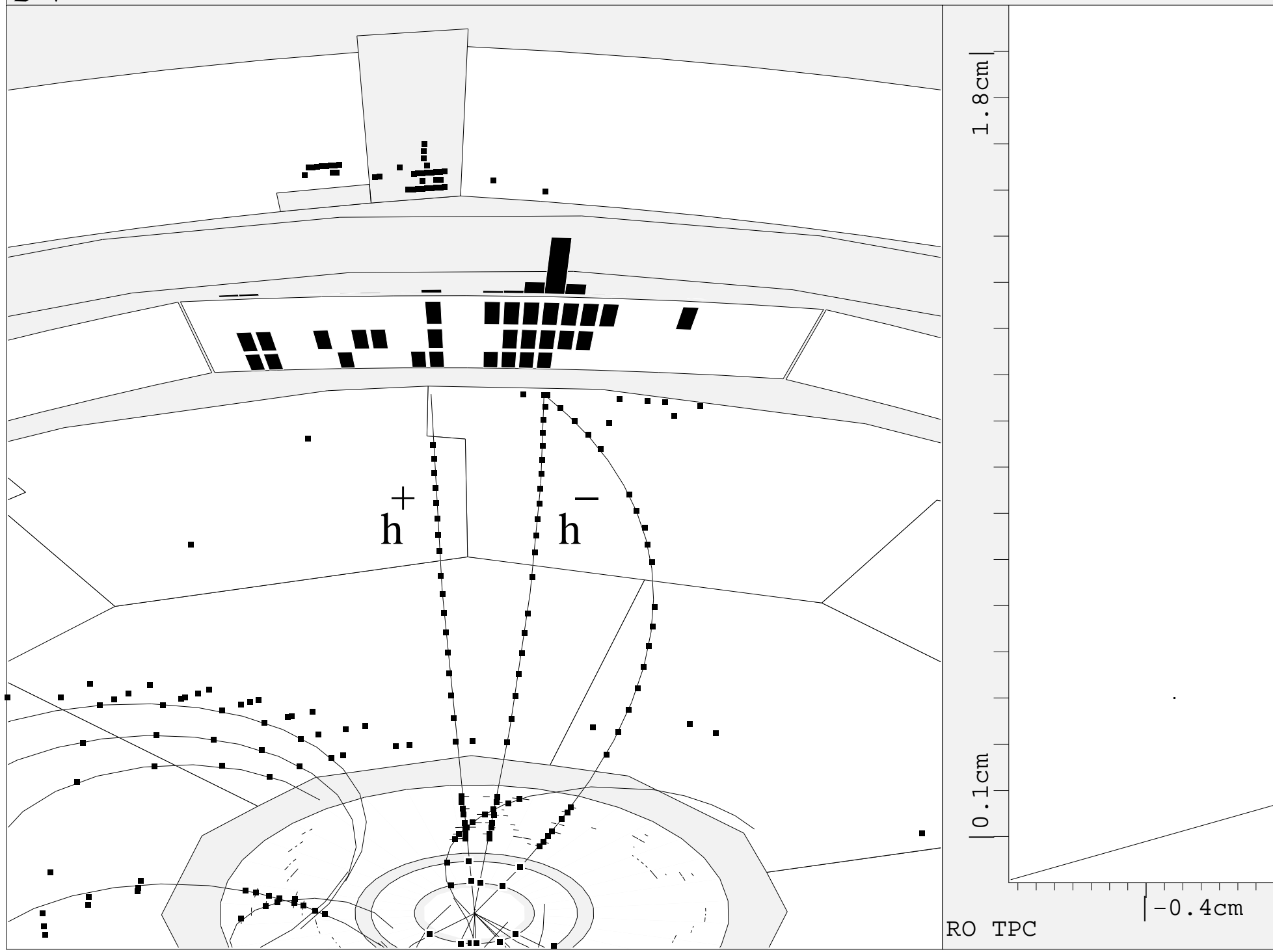

Figure 2: A fish-eye $r \phi$ view of the decay hemisphere and a close-up view of the interaction region for event $b$. The $B$ hadron candidate tracks $\left(h^{+}, h^{-}\right)$form a consistent vertex (B), well displaced from the interaction point (IP). This vertex and the interaction point are indicated together with their three-standard-deviation error ellipses.

to correctly reproduce the efficiency of the vertex cuts was checked using a sample of cleanly reconstructed $\mathrm{J} / \psi \rightarrow \mu^{+} \mu^{-}$decays. The number of selected decays where both tracks have at least one $r \phi$ coordinate and at least one $z$ coordinate in the VDET, and form a consistent vertex, was measured to be $(3 \pm 4) \%$ higher in the data than in the Monte Carlo. The total systematic uncertainty is obtained by adding the contributions from the different sources in quadrature.

An unbinned maximum likelihood fit was performed on the selected event sample in order to extract the average branching ratio $\mathrm{B}_{\mathrm{d}}^{0} \rightarrow \pi^{+} \pi^{-}, \mathrm{B}_{\mathrm{s}}^{0} \rightarrow \pi^{+} \mathrm{K}^{-}$relative to all decays, $R=\left(\mathcal{B} r\left(\mathrm{~B}_{\mathrm{d}}^{0} \rightarrow \pi^{+} \pi^{-}\right)+r_{\mathrm{s}} \mathcal{B} r\left(\mathrm{~B}_{\mathrm{s}}^{0} \rightarrow \pi^{+} \mathrm{K}^{-}\right)\right) /\left(\mathcal{B} r\left(\mathrm{~B}_{\mathrm{d}}^{0} \rightarrow \mathrm{h}^{+} \mathrm{h}^{-}\right)+r_{\mathrm{s}} \mathcal{B} r\left(\mathrm{~B}_{\mathrm{s}}^{0} \rightarrow \mathrm{h}^{+} \mathrm{h}^{-}\right)\right)$, where $r_{\mathrm{s}}$ 

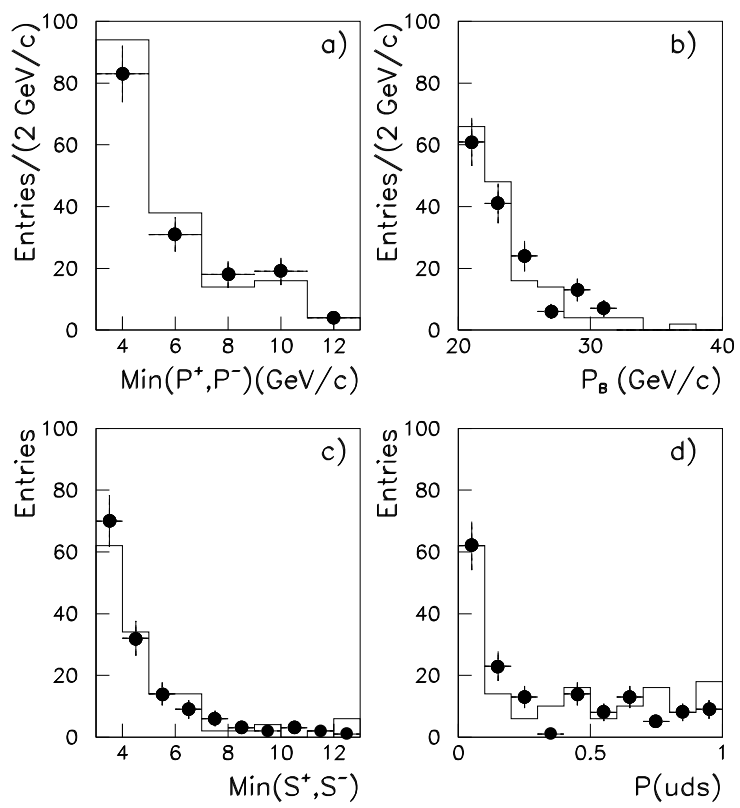

Figure 3: Comparisons between data (points with error bars) and Monte Carlo simulation, normalised to the number of data events (histogram), for the relaxed selection showing: (a) the smaller of the two individual track momenta $\left(P^{+}, P^{-}\right)$; (b) the momentum $P_{B}$ of the $\mathrm{B}$ hadron candidate; (c) the smaller of the two impact parameter significances of the track pair $\left(S^{+}, S^{-}\right)$; (d) the probability $\mathcal{P}_{\text {uds }}$ that all tracks in the hemisphere opposite to the $\mathrm{B}$ hadron candidate originate from the interaction point.

is the ratio of $B_{s}^{0}$ to $B_{d}^{0}$ decays in the sample. The likelihood is defined as

$$
\begin{aligned}
& \mathcal{L}(R)=\prod_{i=1}^{n}\left[\quad R \quad \times\left(f_{\mathrm{d}} \mathcal{P}\left(\mathrm{B}_{\mathrm{d}}^{0} \rightarrow \pi^{+} \pi^{-}\right)+0.5 f_{\mathrm{s}}\left(\mathcal{P}\left(\mathrm{B}_{\mathrm{s}}^{0} \rightarrow \pi^{+} \mathrm{K}^{-}\right)+\mathcal{P}\left(\overline{\mathrm{B}}_{\mathrm{s}}^{0} \rightarrow \mathrm{K}^{+} \pi^{-}\right)\right)\right)+\right. \\
& \left.(1-R) \times\left(0.5 f_{\mathrm{d}}\left(\mathcal{P}\left(\mathrm{B}_{\mathrm{d}}^{0} \rightarrow \mathrm{K}^{+} \pi^{-}\right)+\mathcal{P}\left(\overline{\mathrm{B}}_{\mathrm{d}}^{0} \rightarrow \pi^{+} \mathrm{K}^{-}\right)\right)+f_{\mathrm{s}} \mathcal{P}\left(\mathrm{B}_{\mathrm{s}}^{0} \rightarrow \mathrm{K}^{+} \mathrm{K}^{-}\right)\right)\right]
\end{aligned}
$$

where $f_{\mathrm{d}}$ and $f_{\mathrm{s}}$ are the fractions of $\mathrm{B}_{\mathrm{d}}^{0}$ and $\mathrm{B}_{\mathrm{s}}^{0}$ decays in the sample, respectively, and the probability density $\mathcal{P}$ for any given hypothesis is the product of the three probability densities

Table 2: Relative systematic uncertainties in the branching ratio measurement.

\begin{tabular}{|l||c|}
\hline Lifetimes and hadronization & $2 \%$ \\
Combinatorial background & $1 \%$ \\
Neutral charmless background & $7 \%$ \\
b fragmentation & $3 \%$ \\
Vertex cuts & $4 \%$ \\
\hline \hline Total (in quadrature) & $9 \%$ \\
\hline
\end{tabular}


Table 3: Probability densities of the four candidates.

\begin{tabular}{|c|c|c|c|c|}
\hline Hypothesis & Event a & Event $b$ & Event $\mathrm{c}$ & Event $d$ \\
\hline $\mathcal{P}\left(\mathrm{B}_{\mathbf{d}}^{0} \rightarrow \pi^{+} \pi^{-}\right)$ & 0.0319 & 0.0021 & 0.0445 & 0.0018 \\
\hline $\mathcal{P}\left(\mathrm{B}_{\mathrm{d}}^{0} \rightarrow \mathrm{K}^{+} \pi^{-}\right)+\mathcal{P}\left(\overline{\mathrm{B}}_{\mathrm{d}}^{0} \rightarrow \pi^{+} \mathrm{K}^{-}\right)$ & 0.0009 & 0.0064 & 0.0007 & 0.0011 \\
\hline $\mathcal{P}\left(\mathrm{B}_{\mathrm{s}}^{0} \rightarrow \pi^{+} \mathrm{K}^{-}\right)+\mathcal{P}\left(\overline{\mathrm{B}}_{\mathrm{s}}^{0} \rightarrow \mathrm{K}^{+} \pi^{-}\right)$ & 0.0050 & 0.0217 & 0.0048 & 0.0043 \\
\hline $\mathcal{P}\left(\mathrm{B}_{\mathrm{s}}^{0} \rightarrow \mathrm{K}^{+} \mathrm{K}^{-}\right)$ & 0.0002 & 0.0001 & 0.0003 & 0.0000 \\
\hline $\mathcal{P}\left(\Lambda_{\mathrm{b}} \rightarrow \mathrm{p} \pi^{-}\right)+\mathcal{P}\left(\bar{\Lambda}_{\mathrm{b}} \rightarrow \pi^{+} \overline{\mathrm{p}}\right)$ & 0.0002 & 0.0000 & 0.0000 & 0.0000 \\
\hline $\mathcal{P}\left(\Lambda_{\mathrm{b}} \rightarrow \mathrm{pK}^{-}\right)+\mathcal{P}\left(\bar{\Lambda}_{\mathrm{b}} \rightarrow \mathrm{K}^{+} \overline{\mathrm{p}}\right)$ & 0.0000 & 0.0000 & 0.0000 & 0.0000 \\
\hline
\end{tabular}

Table 4: Relative systematic uncertainties in the ratio of branching ratios.

\begin{tabular}{|l||l|}
\hline Lifetimes and hadronization & $2 \%$ \\
Combinatorial background & $1 \%$ \\
Neutral charmless background & $7 \%$ \\
Non-Gaussian tails & $6 \%$ \\
\hline \hline Total (in quadrature) & $9 \%$ \\
\hline
\end{tabular}

corresponding to the mass and $\mathrm{dE} / \mathrm{dx}$ measurements together with their respective uncertainties. The probability densities of the four candidate events, under various decay hypotheses, are given in Table 3 . The fit has also been performed containing corresponding terms from $\Lambda_{\mathrm{b}}$ decays; however, for the four selected events the $\Lambda_{\mathrm{b}}$ hypothesis is very unlikely and these terms do not change the results. The fractions $f_{\mathrm{d}}$ and $f_{\mathrm{s}}$ were calculated assuming that $\Gamma\left(\mathrm{B}_{\mathrm{d}}^{0} \rightarrow \pi^{+} \pi^{-}\right)=\Gamma\left(\mathrm{B}_{\mathrm{s}}^{0} \rightarrow \pi^{+} \mathrm{K}^{-}\right)$ and $\Gamma\left(\mathrm{B}_{\mathrm{d}}^{0} \rightarrow \mathrm{K}^{+} \pi^{-}\right)=\Gamma\left(\mathrm{B}_{\mathrm{s}}^{0} \rightarrow \mathrm{K}^{+} \mathrm{K}^{-}\right)$and taking into account the different lifetimes and hadronization factors as above. The fit also assumed that the contributions of other charmless $\mathrm{B}$ decays, such as $\mathrm{B}_{\mathrm{d}}^{0} \rightarrow \mathrm{K}^{+} \mathrm{K}^{-}$and $\mathrm{B}_{\mathrm{s}}^{0} \rightarrow \pi^{+} \pi^{-}$, were negligible. The fit yielded

$$
R=1.0_{-0.3}^{+0.0+0.0}+
$$

where the statistical uncertainty quoted corresponds to a region containing $68.4 \%$ of the integrated likelihood. The value of $R$ is greater than 0.4 with a $95 \%$ confidence level.

The sources of systematic uncertainty considered are given in Table 4. The uncertainties in the hadronization of $\mathrm{b}$ quarks into the different $\mathrm{B}$ hadrons and their measured lifetimes give a contribution of $2 \%$. The effect of the small background contributions coming from the combinatorial and charmless neutral backgrounds is conservatively taken as maximal. The effects of the non-Gaussian tails on the mass and $\mathrm{dE} / \mathrm{dx}$ measurements were studied in the data. The $\mathrm{dE} / \mathrm{dx}$ distribution of muons in a selected sample of $\mathrm{J} / \psi \rightarrow \mu^{+} \mu^{-}$decays was fitted to a double Gaussian. The width of the second Gaussian was fixed to be twice that of the first. The fraction of tracks contained in the second Gaussian was measured to be $(2.5 \pm 2.0) \%$. For charmless $\mathrm{B}$ decays passing the above event selection, the uncertainty in the mass measurement is dominated by the contribution coming from the momentum measurement of the individual tracks. The nonGaussian tails of the momentum resolution for high momentum tracks were studied in a sample of selected $\mathrm{Z} \rightarrow \mu^{+} \mu^{-}$decays. The study was restricted to the upper side of the distribution to minimize the effect of initial-state radiation. The fraction of tracks contained in a second Gaussian with double width was measured to be $(5.0 \pm 0.5 \%)$. When the likelihood fit was repeated taking into account these non-Gaussian tails, the lower limit on $R$ decreased by an amount corresponding 
Table 5: Consistency of the candidate events with the different hypotheses expressed as a $\chi^{2}$ probability $\mathcal{P}_{\chi^{2}}$ based on the mass and the $\mathrm{dE} / \mathrm{dx}$ measurements of each track. Also shown are the $90 \%$ confidence level upper limits obtained for the branching ratios under these hypotheses.

\begin{tabular}{|l|c|c|c|c|c|}
\hline Decay Hypothesis & $\begin{array}{c}\text { Event } \\
\mathrm{a}\end{array}$ & $\begin{array}{c}\text { Event } \\
\mathrm{b}\end{array}$ & $\begin{array}{c}\text { Event } \\
\mathrm{c}\end{array}$ & $\begin{array}{c}\text { Event } \\
\mathrm{d}\end{array}$ & $\begin{array}{c}\text { 90\% Confidence Level } \\
\text { BR Upper Limit }\end{array}$ \\
\hline $\mathrm{B}_{\mathrm{d}}^{0} \rightarrow \pi^{+} \boldsymbol{\pi}^{-}$ & $\mathbf{0 . 7 1}$ & $\mathbf{0 . 0 8}$ & $\mathbf{0 . 8 7}$ & $\mathbf{0 . 0 7}$ & $4.1 \times 10^{-5}$ \\
\hline $\mathrm{B}_{\mathrm{d}}^{0} \rightarrow \mathrm{K}^{+} \boldsymbol{\pi}^{-}$ & $\mathbf{0 . 0 6}$ & $\mathbf{0 . 3 6}$ & $\mathbf{0 . 0 4}$ & $\mathbf{0 . 0 8}$ & $3.0 \times 10^{-5}$ \\
\hline $\mathrm{B}_{\mathrm{d}}^{0} \rightarrow \mathrm{K}^{+} \mathrm{K}^{-}$ & $<\mathbf{0 . 0 1}$ & $<\mathbf{0 . 0 1}$ & $<\mathbf{0 . 0 1}$ & $<\mathbf{0 . 0 1}$ & $1.8 \times 10^{-5}$ \\
\hline $\mathrm{B}_{\mathrm{d}}^{0} \rightarrow \mathrm{p} \overline{\mathrm{p}}$ & $<\mathbf{0 . 0 1}$ & $<\mathbf{0 . 0 1}$ & $<\mathbf{0 . 0 1}$ & $<\mathbf{0 . 0 1}$ & $1.8 \times 10^{-5}$ \\
\hline $\mathrm{B}_{\mathrm{s}}^{0} \rightarrow \pi^{+} \pi^{-}$ & $\mathbf{0 . 8 7}$ & $\mathbf{0 . 1 0}$ & $\mathbf{0 . 0 8}$ & $\mathbf{0 . 1 2}$ & $1.7 \times 10^{-4}$ \\
\hline $\mathrm{B}_{\mathrm{s}}^{0} \rightarrow \pi^{+} \mathrm{K}^{-}$ & $\mathbf{0 . 1 8}$ & $\mathbf{0 . 8 6}$ & $\mathbf{0 . 2 8}$ & $\mathbf{0 . 2 6}$ & $2.1 \times 10^{-4}$ \\
\hline $\mathrm{B}_{\mathrm{s}}^{0} \rightarrow \mathrm{K}^{+} \mathrm{K}^{-}$ & $\mathbf{0 . 0 1}$ & $<\mathbf{0 . 0 1}$ & $\mathbf{0 . 0 1}$ & $<\mathbf{0 . 0 1}$ & $5.9 \times 10^{-5}$ \\
\hline $\mathrm{B}_{\mathrm{s}}^{0} \rightarrow \mathrm{p} \overline{\mathrm{p}}$ & $<\mathbf{0 . 0 1}$ & $<\mathbf{0 . 0 1}$ & $<\mathbf{0 . 0 1}$ & $<\mathbf{0 . 0 1}$ & $5.9 \times 10^{-5}$ \\
\hline$\Lambda_{\mathrm{b}} \rightarrow \mathrm{p} \boldsymbol{\pi}^{-}$ & $\mathbf{0 . 0 1}$ & $<\mathbf{0 . 0 1}$ & $<\mathbf{0 . 0 1}$ & $<\mathbf{0 . 0 1}$ & $5.0 \times 10^{-5}$ \\
\hline$\Lambda_{\mathrm{b}} \rightarrow \mathrm{pK} \mathrm{K}^{-}$ & $<\mathbf{0 . 0 1}$ & $<\mathbf{0 . 0 1}$ & $<\mathbf{0 . 0 1}$ & $<\mathbf{0 . 0 1}$ & $5.0 \times 10^{-\mathbf{5}}$ \\
\hline
\end{tabular}

to a contribution added in quadrature of $6 \%$. The total systematic uncertainty is obtained by adding the contributions from the different sources in quadrature.

\section{Upper limits on exclusive final states}

The selected event sample is also used to set upper limits for the branching ratios of exclusive charmless hadronic two-body decays of B hadrons. For each decay hypothesis a probability $\mathcal{P}_{\chi^{2}}$ is obtained from the measured mass of the system and the $\mathrm{dE} / \mathrm{dx}$ values of the two tracks. From the total number of events consistent with each final state, having $\mathcal{P}_{\chi^{2}}>10 \%$, the $90 \%$ confidence level upper limits for the individual branching ratios are calculated and given in Table 5 . This calculation assumes that $\Gamma(\mathrm{Z} \rightarrow \mathrm{b} \overline{\mathrm{b}}) / \Gamma(\mathrm{Z} \rightarrow \mathrm{q} \overline{\mathrm{q}})=0.22$ and that the fractions of $\mathrm{b}$ quarks hadronizing into $\mathrm{B}_{\mathrm{d}}^{0}, \mathrm{~B}_{\mathrm{s}}^{0}$ and $\Lambda_{\mathrm{b}}$ are as given above. The cut on $\mathcal{P}_{\chi^{2}}$ was optimized to give the lowest average branching ratio upper limit for a sample of Monte Carlo experiments generated without signal production using the technique described in Ref. [19]. To be conservative no background subtraction is performed in deriving these upper limits. The branching ratio upper limits obtained are competitive with those obtained elsewhere $[4,5,6]$ but are still at least a factor of two larger than theoretical expectations $[1,2,17]$.

\section{Conclusions}

A search for charmless hadronic B decays has been performed in data recorded by the ALEPH detector at LEP. Four candidate events are observed in a sample of four million hadronic $\mathrm{Z}$ decays. The probability that these events come from background sources is estimated to be less than $10^{-6}$. The average branching ratio of weakly decaying $B$ hadrons into two long-lived charged hadrons (pions, kaons or protons), weighted by their production cross sections and lifetimes, is measured to be $\mathcal{B} r\left(\mathrm{~B} \rightarrow \mathrm{h}^{+} \mathrm{h}^{-}\right)=\left(1.7_{-0.7}^{+1.0} \pm 0.2\right) \times 10^{-5}$. The relative branching fraction $R$ $=\left(\mathcal{B} r\left(\mathrm{~B}_{\mathrm{d}}^{0} \rightarrow \pi^{+} \pi^{-}\right)+r_{\mathrm{s}} \mathcal{B} r\left(\mathrm{~B}_{\mathrm{s}}^{0} \rightarrow \pi^{+} \mathrm{K}^{-}\right)\right) /\left(\mathcal{B} r\left(\mathrm{~B}_{\mathrm{d}}^{0} \rightarrow \mathrm{h}^{+} \mathrm{h}^{-}\right)+r_{\mathrm{s}} \mathcal{B} r\left(\mathrm{~B}_{\mathrm{s}}^{0} \rightarrow \mathrm{h}^{+} \mathrm{h}^{-}\right)\right)$, where $r_{\mathrm{s}}$ is the ratio of $\mathrm{B}_{\mathrm{s}}^{0}$ to $\mathrm{B}_{\mathrm{d}}^{0}$ decays in the sample, is measured from an unbinned maximum likelihood fit 
to be $R=1.0_{-0.3}^{+0.0}+0.0$. In addition, branching ratio upper limits of order $10^{-4}$ are obtained for a variety of exclusive charmless hadronic two-body decays of $B$ hadrons.

\section{Acknowledgements}

We wish to thank our colleagues from the accelerator divisions for the successful operation of LEP. We are indebted to the engineers and technicians at CERN and our home institutes for their contribution to the good performance of ALEPH. Those of us from non-member countries thank CERN for its hospitality. 


\section{References}

[1] M. Gronau, Phys. Lett. B300 (1993) 163.

[2] A. Deandrea et al., Phys. Lett. B320 (1994) 170.

[3] S. Stone (CLEO Collaboration), Nucl. Instrum. Methods A368 (1995) 68;

J. Haba (BELLE Collaboration), Nucl. Instrum. Methods A368 (1995) 74;

P.F. Harrison (BABAR Collaboration), Nucl. Instrum. Methods A368 (1995) 81;

A. Nisati (ATLAS Collaboration), Nucl. Instrum. Methods A368 (1995) 109;

D. Kotlinski and C. Racca (CMS Collaboration), Nucl. Instrum. Methods A368 (1995) 115;

W. Schmidt-Parzefall (HERA-B Collaboration), Nucl. Instrum. Methods A368 (1995) 124;

S. Erhan (LHC-B Collaboration), Nucl. Instrum. Methods A368 (1995) 133.

[4] CLEO Collaboration, M. Battle et al., Phys. Rev. Lett. 71 (1993) 3922;

CLEO Collaboration, D.M. Asner et al., CLNS-95-1338.

[5] OPAL Collaboration, R. Akers et al., Phys. Lett. B337 (1994) 393.

[6] DELPHI Collaboration, P. Abreu et al., "Study of rare b decays with the DELPHI detector at LEP", CERN-PPE/96-067, submitted to Z. Phys. C.

[7] ALEPH Collaboration, D. Decamp et al., Nucl. Instrum. Methods A294 (1990) 121.

[8] ALEPH Collaboration, D. Buskulic et al., Nucl. Instrum. Methods A360 (1995) 481.

[9] ALEPH Collaboration, D. Decamp et al., Z. Phys. C53 (1992) 1.

[10] ALEPH Collaboration, D. Buskulic et al., Phys. Lett. B295 (1992) 174.

[11] ALEPH Collaboration, D. Buskulic et al., Nucl. Instrum. Methods A346 (1994) 461.

[12] G. Bauer (CDF Collaboration), "B-Physics at CDF", Proceedings of the $2^{\text {nd }}$ Rencontres du Vietnam, Oct 1995;

DELPHI Collaboration, P. Abreu et al., Phys. Lett. B374 (1996) 351;

ALEPH Collaboration, D. Buskulic et al., "Measurement of the Mass of the $\Lambda_{\mathrm{b}}$ Baryon", CERN-PPE/96-028, to be published in Phys. Lett. B.

[13] ALEPH Collaboration, D. Buskulic et al., "Measurement of the b forward-backward asymmetry and mixing using high $p_{\perp}$ leptons", CERN-PPE/96-072, submitted to Phys. Lett. B.

[14] ALEPH Collaboration, D. Buskulic et al., Phys. Lett. B313 (1993) 535.

[15] H.-G. Moser, "B-Lifetimes at LEP", to be published in the proceedings of the International Europhysics Conference on High Energy Physics, Brussels, Belgium, July 1995.

[16] R. M. Barnett et al., "Review of Particle Physics, 1996 Edition", Phys. Rev. D 54 (1996) to be published.

[17] A. Deandrea et al., Phys. Lett. B318 (1993) 549.

[18] ALEPH Collaboration, D. Buskulic et al., Phys. Lett. B357 (1995) 699.

[19] ALEPH Collaboration, D. Buskulic et al., Phys. Lett. B313 (1993) 299. 